\title{
Evaluation of Biofilm Formation by Three Different Methods and its Antibiogram with Special Reference to Indwelling Medical Devices from a Tertiary Care Hospital
}

\author{
Sufia Siddhiqui, Uzma Afreen and Santosh Kotgire*
}

Department of Microbiology, Indian Institute of Medical Sciences \& Research, Warudi, Jalna, Maharashtra (India)

\begin{abstract}
Backgound: Biofilms represent the exopolysaccharides produced by the bacteria on various indwelling devices in which they remain enmeshed. These bacteria are highly resistant to antimicrobial agents causing chronic and recurrent infections. So the present study was undertaken to detect biofilms from clinical isolates by three different methods with its antibiotic resistance pattern and association with various indwelling devices.

Material and Methods: The study was carried out in the Department of Microbiology, Indian Institute of Medical Science and Research Jalna over a period of three months. This is a cross-sectional type of observational study. A total of 112 clinical isolates were first identified by standard microbiological tests and then screened for biofilm formation by 1)Tube method,2)Tissue culture method and 3) Congo red agar method. Their antibiotic sensitivity pattern was determined using Kirby Bauer Disc Diffusion method and association with indwelling medical devices was observed prospectively\& retrospectively.

Results: Out of the 112 clinical isolates,we found 57(50.9\%) isolates produced biofilm by tissue culture method,33(29.46\%) by tube method and only16(14.25\%) by Congo red agar method.The predominant biofilm producer was Pseudomonas(70\%) followed by Staphylococcus aureus(61.1\%), Klebsiella (45.83\%),Coagulase negative Staphylococcus(42.85\%)and then E coli(32.14\%).All the biofilm producing strains were highly resistant to commonly used antibiotics and there was a strong association between biofilm production and indwelling medical devices used for the diagnostic or therapeutic intervention.

Conclusions: Tissue culture method was the most sensitive method for detection of biofilms. As the resistance of these isolates was very high, all the isolates from the medical devices should be screened both for biofilm production and their antibiotic sensitivity testing should be performed for better patients compliance and outcome.
\end{abstract}

Keywords: Biofilms, Enterococcus, Staphylococcus, Staphylococcus

\section{Introduction}

Microorganisms have the potential to live in one of the two phenotypes: sessile or planktonic. Planktonic are free floating microorganisms. ${ }^{[1]}$ Sessile phenotype results from attachment to solid surfaces, their irreversible binding and development of exopolysaccharide with gradual increase in the thickness of cellular exudates. Biofilms represent these cellular aggregates enmeshed within the exopolysaccharide matrix. ${ }^{[2]}$

Various indwelling medical devices like urinary catheter, endotracheal tubes, intravascular catheters, artificial joints etc., act as the nidus for the formation of these biofilms. In addition, microorganisms enmeshed in these biofilms evade antimicrobial challenges by multiple mechanisms. ${ }^{[3]}$ These mechanisms include 1) Failure of an agent to penetrate the full depth of the film. 2) Some of the cells in a biofilm experience nutrient limitation and therefore exist in a slow growing or starved state making them not very susceptible to many antimicrobial agents.3) Some of the cells in a biofilms adopt a distinct and protected biofilm phenotype which is biologically programmed response to growth on a surface.

Biofilms are produced by both Gram-positive and Gramnegative bacteria. Enterococcus faecalis, Staphylococcus aureus, Staphylococcus epidermidis, Streptococcus viridans, Escherichia coli, Klebsiella pneumoniae, Proteus mirabilis and Pseudomonas aeruginosa are commonly associated with the production of biofilms. ${ }^{[4]}$

The expression of biofilms in these bacteria is mainly determined by operons. Operons are the clusters of coregulated genes with related functions. The series of genes in an operon are transcribed as a single mRNA and consists of an upstream promoter and a downstream terminator. ${ }^{[5]}$ The biofilm production in Staphylococcus spp is regulated by the expression of polysaccharide intracellular adhesion (PIA) which is the gene product of 
operon icaADBC. ${ }^{[6]}$ In Pseudomonas aeruginosa, various operons that code for alginate, polysaccharides (psl and pel), proteins, cyclic-di-GMP-regulated adhesin A protein $(c d r A)$, cup fimbria, type IV pili, lectins and extracellular DNA (eDNA) have been elucidated for the production of extracellular polysaccharide. ${ }^{[7]}$

With the advancement in medical fields, the use of indwelling medical devices is on the rise which further facilitates biofilm formation and chronicity of infection. There are various methods for biofilm detection which include Tissue culture plate method, Tube method, Congo red agar method, bioluminescent assay and light or fluorescent microscopic examination. The information of biofilm production would help the clinician to evaluate the measure of its virulence and then decide the line of treatment. ${ }^{[8,9]}$ So the present study was undertaken to follow-up all the clinical isolates in our hospital for biofilm production, to assess their antibiotic sensitivity pattern and to find out their association with indwelling medical devices.

\section{Materials and methods}

Setting and Design: The study was carried out in the Department of Microbiology of Indian Institute of Medical science and Research ,Warudi, Jalna for a period of three months from July 2016 to September 2016

Inclusion Criteria: After obtaining clearance from the ethical committee of the Instititue, all the patients admitted to the hospital were included in the study. The samples that were sent to microbiology department included pus, sputum, urine ,blood ,body fluids like pleural fluid, ascitic fluid etc. The isolates were identified by standard microbiological techniques (Gram stain,motility,culture on blood agar, MacConkeys agar, and biochemical identification). ${ }^{[10,11]}$

The samples that showed positive growth were then analysed prospectively and retrospectively for the use of any indwelling medical device in each of these patients.

Bacterial Strains: A total of 112 clinical isolates were subjected to biofilm detection methods. As a positive biofilm producer,we used Staphylococcus epidermidis ATCC 35984 and Staphylococcus aureus ATCC 35556, as the reference strain. Pseudomonas aeruginosa ATCC 27853, Escherichia coli ATCC 35218 and Staphylococcus epidermidis ATCC 12228 (non-slime producer) were used as control.

Detection of Biofilm Formation: All the isolates were subjected to biofilm production by the three methods.

Tube Method (TM): A qualitative assessment of biofilm formation was done, as previously proposed by
Christensen et al. ${ }^{[9]}$ A loopful of microorganism from overnight culture plates were inoculated in Trypticase Soya Broth with glucose $(10 \mathrm{~mL})$ and incubated for 24 hours at $37^{\circ} \mathrm{C}$. The tubes were decanted and washed with Phosphate Buffer Saline $(\mathrm{pH} 7.3)$ and dried.They were then stained with crystal violet $(0.1 \%)$. Excess stain was removed and they were then washed with deionized water. Tubes were again dried in inverted position and observed for biofilm formation.

When a visible film lined the wall and bottom of the tube, biofilm formation was considered positive. If there was a ring formation at the liquid interface, it was not indicative of biofilm formation. The tubes were examined for biofilm formation and the amount of biofilm formation was scored as 0 -absent, 1-weak, 2-moderate or 3-strong. The experiment was done in triplicate and repeated thrice.

Congo Red Agar Method.(CRA) A simple qualitative method to detect biofilm production by using Congo Red Agar (CRA) medium was described by Freeman et al. ${ }^{[12]}$ CRA medium was prepared using brain heart infusion broth $37 \mathrm{~g} / \mathrm{L}$, sucrose $50 \mathrm{~g} / \mathrm{L}$, agar No. 1, $10 \mathrm{~g} / \mathrm{L}$ and Congo Red indicator $8 \mathrm{~g} / \mathrm{L}$. First Congo Red stain was prepared as a concentrated aqueous solution and autoclaved $\left(121^{\circ} \mathrm{C}\right.$ for 15 minutes) separately from the other medium constituents and it was then added to the autoclaved brain heart infusion agar with sucrose at $55^{\circ} \mathrm{C} .{ }^{[13]}$ Test organisms were inoculated on Congo Red Agar plates and incubated at $37^{\circ} \mathrm{C}$ for $24 \mathrm{~h}$ aerobically. Black colonies with a dry crystalline consistency indicated biofilm production. The experiment was performed in triplicate and repeated three times.

Tissue Culture Plate (TCP) Method: Overnight broth cultures of bacteria in Trypticase soya broth (TSB) with $1 \%$ glucose were diluted $1: 100.200 \mu 1$ portions were inoculated in 96 well flat bottom polystyrene microtitre plates ( Nuclon TM Ltd ). ${ }^{[14]}$ Microtitre plates were incubated at $35{ }^{\circ} \mathrm{C}$ for 24 hours. Cultures were then aspirated from these wells and the wells were washed 3 times with phosphate buffer saline at $\mathrm{pH}$ 7.2. The plates were then air dried overnight and stained with $0.1 \%$ crystal violet. The optical density of the wells was measured at 490 $\mathrm{nm}$ using micro ELISA auto reader. (Robonic- Readwell touch-Automatic ELISA plate analyser).An optical density of 0.12 was considered as positive to distinguish biofilm producers from those that did not form biofilm. Sterile TSB was used as a negative control (Blank). Intensity of Biofilm was classified as given by Mathur et al. ${ }^{[6]}$ (Table 1)

Antibiotic Sensitivity Testing: Antibiotic sensitivity testing was performed as per CLSI guidelines ${ }^{[15]}$ using Kirby Bauer's Disc diffusion method on Mueller Hinton 
Agar plate with the following antibiotics. Ofloxacin $(5 \mu \mathrm{g})$, Cefoxitin $(30 \mu \mathrm{g})$, Cefotazidime \& Clavulonic acid $(30 / 10 \mu \mathrm{g})$, Erythromycin $(15 \mu \mathrm{g})$, Cefipime $(30 \mu \mathrm{g})$, Cotrimoxazole $(25 \mu \mathrm{g})$, Amikacin $(30 \mu \mathrm{g})$, Gentamicin $(10 \mu \mathrm{g})$, Nitrofurantoin $(30 \mu \mathrm{g})$, Linezolid $(30 \mu \mathrm{g})$, Vancomycin $(30 \mu \mathrm{g})$, Imipenem $(10 \mu \mathrm{g})$, Pipercillin \&Tazobactum $(100 / 10 \mu \mathrm{g})$, and Colistin $(10 \mu \mathrm{g})$. Antibiotics discs were procured from HiMedia Laboratories Pvt. Ltd, India.

\section{Results}

Out of 112 isolates, tissue culture plate showed the maximum $57(50.9 \%)$ isolates positive for biofilm production, of which $20(17.85 \%)$ showed high biofilm production and $37(33.04 \%)$ showed moderate biofilm production. (Table 2).Tube method showed biofilm production in 33 (29.46\%) isolates among which $12(10.71 \%)$ showed high biofilm production and 21(18.75\%) showed moderate biofilm production. Biofilm production in Congo red agar method was seen in only $16(14.28 \%)$ of isolates.

On statistical analysis, the sensitivity and positive predictive value of tube method was more than that of Congo red agar method although the specificity of both remained the same. (Table 3)

Of all the isolates, Pseudomonas aeruginosa showed maximum biofilm production $70 \%$, followed by Staphylococcus aureus (59.46\%), Klebsiella spp (44\%),
Staphylococcus epidermidis (42.85\%), Lastly E coli showed biofilm production in only $(32.14 \%)$ of isolates. (Table 4).

Maximum association between biofilm production and indwelling medical devices was observed with Staphylococcus aureus (40\%)

When the resistance pattern of biofilm producing isolates was seen, it was found that in Gram positive bacteria, $72.73 \%$ of Staphylococcus aureus, and $66.66 \%$ of Coagulase negative Staphylococcus were resistant to Erythromycin. (Table 5) Similar pattern of high resistance was observed to Cotrimoxazole (63.64\% in Staphylococcus aureus and $100 \%$ in Coagulase negative Staphylococcus) Cefoxitin is considered as the surrogate marker for Methicillin resistant Staphylococcus ${ }^{[15]}$.In our study more than $60 \%$ of the strains were found to be Methicillin resistant(as they were resistant to Cefoxitin).

In gram negative bacteria, Pseudomonas aeruginosa isolated from the indwelling devices showed a very high resistance to Amikacin (71.43\%) and Cefipime. Of the nine strains of $E$ coli that were biofilm producing, (71.43\%) were resistant to Cefipime and 6 strains (66.67\%)were resistant to Gentamicin, Ceftazidime\& Clavulonic acid, and Cotrimoxazole.

Table 1: Classification of bacterial adherence by TCP method.

\begin{tabular}{|l|c|c|}
\hline Mean OD values & Adherence & Biofilm formation \\
\hline$<0.120$ & Non & Non / weak \\
\hline $0.120-0.240$ & Moderately & Moderate \\
\hline$>0.240$ & Strong & High \\
\hline
\end{tabular}

Table 2: Bio film production in the clinical isolate by each of the methods.

\begin{tabular}{|l|c|c|c|}
\hline Bio film formation & TCP & TM & CRA \\
\hline High & $20(17.85 \%)$ & $12(10.71 \%)$ & $05(4.46 \%)$ \\
\hline Moderate & $37(33.04 \%)$ & $21(18.75 \%)$ & $11(9.82 \%)$ \\
\hline Weak / none & $55(49.11 \%)$ & $79(70.54 \%)$ & $96(85.71 \%)$ \\
\hline
\end{tabular}

Table 3: Statistical evaluation of TM and CRA methods for detection of bio film formation in clinical isolates ( $\mathrm{n}=112$ ) taking tissue culture as the gold standard.

\begin{tabular}{|l|c|c|c|c|}
\hline Screening methods & Sensitivity & Specificity & $\begin{array}{c}\text { Positive predictive } \\
\text { value }\end{array}$ & $\begin{array}{c}\text { Negative predictive } \\
\text { value }\end{array}$ \\
\hline Tube method & $57.89 \%$ & $92.73 \%$ & $89.19 \%$ & $68.00 \%$ \\
\hline $\begin{array}{l}\text { Congo red agar } \\
\text { method }\end{array}$ & $28.07 \%$ & $92.73 \%$ & $80 \%$ & $55.43 \%$ \\
\hline
\end{tabular}

Table 4: Correlation of bio film production with organisms isolated $(n=112)$.

\begin{tabular}{|l|c|c|}
\hline Organisms & No. Of isolates $n=112$ & Bio film production in the isolate by either of the methods \\
\hline Pseudomonas aeruginosa & 10 & $7(70 \%)$ \\
\hline Stapylococcus. aureus & 37 & $22(59.46 \%)$ \\
\hline
\end{tabular}




\begin{tabular}{|l|c|c|}
\hline Organisms & No. Of isolates $n=112$ & Bio film production in the isolate by either of the methods \\
\hline Klebsiella spp & 25 & $11(44 \%)$ \\
\hline Staphylococcus epidermidis & 7 & $3(42.85 \%)$ \\
\hline E. coli & 28 & $9(32.14 \%)$ \\
\hline Acinetobacter spp & 2 & 0 \\
\hline Enterococcus spp & 1 & 0 \\
\hline Candida species & 2 & 0 \\
\hline
\end{tabular}

Table 5: Resistance pattern of the bio film producing isolates.

\begin{tabular}{|c|c|c|c|c|c|c|}
\hline \multirow[t]{2}{*}{ Antibiotics } & \multicolumn{2}{|c|}{ Gram positive } & \multirow[t]{2}{*}{ Antibiotics } & \multicolumn{3}{|c|}{ Gram negative } \\
\hline & \begin{tabular}{|c|} 
Staphylococcus \\
aureus \\
$\mathrm{n}=\mathbf{2 2}(\%)$
\end{tabular} & $\begin{array}{l}\text { CONS } \\
n=3(\%)\end{array}$ & & $\begin{array}{l}\text { E. coli } \\
n=9(\%)\end{array}$ & $\begin{array}{c}\text { Klebsiella } \\
\text { spp } n=11(\%)\end{array}$ & $\begin{array}{c}\text { Pseudomonas } \\
\text { aeruginosa } \\
n=7(\%)\end{array}$ \\
\hline Erythromycin & $16(72.73)$ & $2(66.67)$ & Gentamicin & $6(66.67)$ & $1(9.09)$ & $2(28.57)$ \\
\hline Cotrimoxazole & 14(63.64) & $3(100.00)$ & Amikacin & $2(22.22)$ & $2(18.18)$ & $5(71.43)$ \\
\hline Cefoxitin & $14(63.64)$ & $2(66.67)$ & Cefepime & $7(77.78)$ & $5(45.45)$ & $5(71.43)$ \\
\hline Gentamicin & $9(40.91)$ & $1(33.33)$ & Ceftazidime+ Clavulonic acid & $6(66.67)$ & $2(18.18)$ & 0.00 \\
\hline Linezolid & 0.00 & 0.00 & Ofloxacin & 0.00 & 0.00 & $1(14.29)$ \\
\hline Clindamycin & $5(22.73)$ & 0.00 & Co-trimoxazole & $6(66.67)$ & $4(36.36)$ & Not tested \\
\hline Nitrofurantoin & 0.00 & $1(33.33)$ & Imipenem & Not tested & Not tested & $1(14.29)$ \\
\hline \multirow[t]{2}{*}{ Vancomycin } & 0.00 & 0.00 & Piperacillin + Tazobactum & Not tested & Not tested & $3(42.86)$ \\
\hline & & & Colistin & Not tested & Not tested & $3(42.86)$ \\
\hline
\end{tabular}

\section{Bio film producing Isolate Associated with Medical Devices}

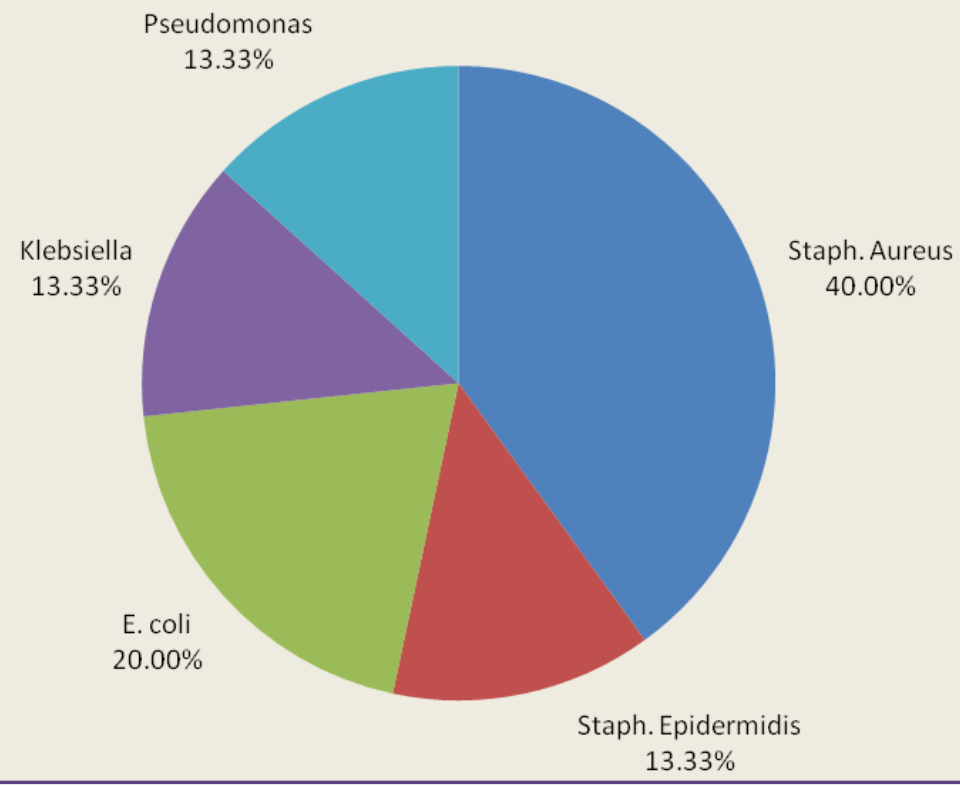

Fig. 1: Correlation of bio film producing organisms isolated and medical devices. 


\section{Discussion}

Biofilms represent an important defense mechanism for microorganisms on indwelling medical devices which enables them to evade the antibiotics so that they can survive for longer period of time. We screened a total of 112 isolates from our hospital for biofilm production by either of the three methods i.e. Tissue culture plate method. Tube method,Congo red agar method.

Tissue culture plate showed biofilm production in $50.9 \%$ of isolates of which strong biofilm production was found in $17.85 \%$ of isolates and moderate biofilm production was seen in $33.05 \%$ of isolates. Mathur et al ${ }^{[6]}$ have reported strong biofilm production in $14.4 \%$ of isolates and moderate biofilm production in $39.4 \%$ while Nabajit deka ${ }^{[17]}$ have reported strong biofilm production in $36 \%$ and moderate biofilm production in $47 \%$ of isolates. The production of biofilm in our isolates was less compared to these findings which could be because of either of the two reasons ,firstly the incubation period we used, for this method was $18 \mathrm{hrs}$ and secondly the absorbance we had used for ELISA was $490 \mathrm{~mm}$. The biofilm production is enhanced in presence of glucose and if the incubation period was increased further. ${ }^{[6]}$ Tube method correlated best with the findings of Tissue culture plate method. Of the $29.46 \%$ of biofilm producing isolates , $10.71 \%$ showed high biofilm production and $18.75 \%$ showed moderate biofilm production but this was again subjected to interobserver variation. Bose et al ${ }^{[18]}$ have reported biofilm production by tube method in $42.46 \%$ of isolates while Mathur et al ${ }^{[6]}$ have reported biofilm production in $41 \%$ of isolates respectively. Congo red agar method was found to be the least sensitive with only $14.28 \%$ of isolates showing biofilm formation .Only $5(4.46 \%)$ isolates produced black coloured colonies while the rest $11(9.82 \%)$ showed pink coloured colonies with crystalline consistency so they were considered as moderately positive. Hasan et al [19] have reported biofilm production by Congo red in $9.9 \%$ of isolates and Nabajit deka ${ }^{[17]}$ in $20 \%$ of isolates. Our findings lie in between the two.

Statistically we found that if tissue culture method was used as a gold standard, Tube method had its sensitivity and positive predictive value more than Congo red agar method which corresponded with the findings of Mathur et al. ${ }^{[6]}$ Most of the Pseudomonas aeruginosa isolates (70 $\%)$ produced biofilms and the resistance they showed to antibiotics varied from 14\% (Ofloxacin and Imipenem) to $71.43 \%$ for (Cefipime \& Gentamicin) Hyderi et al ${ }^{[20]}$ have reported $43.5 \%$ of Pseudomonas aeuginosa producing biofilms in burns patients also Perez at al [21] have done a study on cystic fibrosis patients and found that $71.4 \%$ of cystic fibrosis patients produced biofilms. Staphylococcus species was next isolate which produced maximum biofilms. We found $59.46 \%$ of Staphylococcus aureus strains and $42.85 \%$ of Coagulase negative strains producing biofilms in our study. Sharvari et al [22] reported $45.6 \%$ of Staphylococcus aureus and 38.7\% of Coagulase negative Staphylocoocus producing biofilms. Also Mathur et al ${ }^{[6]}$ and Taj et al ${ }^{[8]}$ have reported biofilms in $57.8 \%$ and $54.78 \%$ of Staphylococcus isolates. Of all the Staphylococcus isolates that produced biofilms, $40 \%$ were associated with indwelling medical devices . Kloos et al ${ }^{[23]}$ have reported Staphylococcus as the predominant species isolated on polymeric devices, their major pathogenic factor being ability to form biofilms on these surfaces. $44 \%$ of Klebsiella spp strains produced biofilms by either of the methods. Seifi et al ${ }^{[24]}$ have shown fully established biofilm production in $33 \%$ of Klebsiella pneumonia and moderate biofilm production in $52.1 \%$ strains of Klebsiella pneumonia.

$32.14 \%$ of $E$ coli strains in our study revealed biofilm production while the study done by Suman et al ${ }^{[25]}$ had revealed $92 \%$ of E coli producing biofilms in uropathogens. Our incidence is less because we had isolated $E$ coli from all possible samples and not restricted ourselves to urinary isolates.

Almost all the isolates showed resistance to antibiotics implying the chronic nature of these infections. Our findings of drug resistance corresponded with that of Sundaram et al ${ }^{[26]}$

\section{Conclusion}

Tube method can be used as an important screening method for detection of biofilms in resource poor settings. If facilities are available, then tissue culture method can be the method of choice. As biofilm producing strains isolated from indwelling medical devices are resistant to commonly used antibiotics, all such biofilm producing strains should be subjected to antibiotic sensitivity testing for better patient management and compliance.

\section{References}

1. John G.T., Donale C.L. Biofilms: architects of disease. In: Connie R.M., Donald C.L., George M., editors. Textbook of diagnostic microbiology. 3rd ed. Saunders 2007 . 884-95.

2. Aparna MS, Yadav S. Biofilms: microbes and disease. Brazilian J of Infect Diseases.2008;12(6):526-30.

3. Costerton J.W., Stewart P.S., Greenberg E.P. Bacterial biofilms: A common cause of persistent infections. Science 1999;284 (5418):1318-22.

4. Donlan RM. Biofilms and device-associated infections. Emerg Infect Dis 2001; 7(2):277-81. 
5. Brouwer RW, Kuipers OP, van Hijum S. The relative value of operon predictions. Brief Bioinform 2008;9: 367-75.

6. Mathur T, Singhal S, Khan S, Upadhyay DJ, Fatma T, Rattan A.Detection of biofilm formation among the clinical isolates of Staphylococci: An evaluation of three different screening methods. Indian J Med Microbiol 2006; 24(1):25-9.

7. Halebeedu PP, GS Vijay K, Shubha G .Revamping the role of biofilm regulating operons in device associated Staphylococci and Pseudomonas aeruginosa. Indian J Med Micro.2014;32(2):112-123.

8. Yasmeen T, Farhan E, Faisal A. Study on biofilm-forming properties of clinical isolates of Staphylococcus aureus. J Infect Dev Ctries 2012; 5(6):403-409.

9. Christensen GD, Simpson WA, Bisno AL, Beachey EH. Adherence of slime-producing strains of Staphylococcus epidermidis to smooth surfaces. Infect Immun 1982;37 :318-26.

10. Koneman EW, Allen SD, Janda WM, Schreckember PC, Winn WC. Koneman's Colour Atlas and text book of Diagnostic Microbiology. 6th edition. Newyork: Lippincott: 2006.97-99.

11. Forbes BA, Sahm DF, Weissfeld AS. In: Bailey and Scott's Diagnostic Microbiology. Mosby Elsevier;2007: 779.

12. Freeman J, Falkiner FR, Keane CT. New method for detecting slime production by coagulase negative staphylococci. J Clin Pathol 1989;42:872-4.

13. Reid G. Biofilms in infectious disease and on medical devices. Int. J. Antimic Ag 1999;11: 223-6.

14. Eftekhar F, Speert DP, Biofilm formation by persistent and non-persistent isolates Staphylococcus epidermidis from a neonatal intensive care unit, J Hosp Infect.2009; 71: 112-116

15. CLSI - Clinical and Laboratory Standards Institute 2016. Performance standards for antimicrobial susceptibility testing. Twenty-second informational supplement. Wayne, PA, USA. CLSI;2016.

16. Bauer AW, Kirby WMM, Sherries JC,Jurek M, Antibiotic susceptibility testing by a standardized single method. American Journal of Clinical Pathology. 1966;45: 493-496
17. Nabajit D .Comparison of Tissue Culture plate method, Tube Method and Congo Red Agar Method for the detection of biofilm formation by Coagulase Negative Staphylococcus isolated from Non-clinical Isolates. Int.J. Curr.Microbiol. App.Sci 2014; 3(10) 810-815

18. Bose S, Khodke M, Basak S, Mallick SK, Detection of Biofilm producing staphylococci; Need of the hour. Journal of Clinical and Diagnostic Research. 2009;3(6):1915-1920.

19. Afreenish H, Javaid U ,Fatima K ,Maria O, Ali Khalid Muhammad Iqbal, Evaluation of different detection methods of biofilm formation in the clinical isolates Braz $\mathrm{J}$ Infect Dis . 2011 Vol.15 (4):305-311

20. Samira Heydari 1; Fereshteh Eftekhar 1, Biofilm Formation and $\beta$-Lactamase Production in Burn Isolates of Pseudomonas aeruginosa Jundishapur J Microbiol. 2015 March; 8(3):1-5

21. Perez, L.R.R, Costa, M.C.N.2; Freitas, A.L.P.2; Barth A.L.2,3 Evaluation Of Biofilm Production By Pseudomonas Aeruginosa Isolates Recovered From Cystic Fibrosis And Non-Cystic Fibrosis Patients Brazilian Journal of Microbiology (2011) 42: 476-479.

22. Sharvari AS, Pai Chitra G .Evaluation Of Different Detection Methods Of Biofilm Formation In Clinical Isolates Of Staphylococci. Int J Pharm Bio Sci 2012 Oct; 3(4): (B) $724-733$.

23. Kloos WE, Bannerman TL. Update on clinical significance of coagulase-negative Staphylococci. Clin Microbiol Rev 1994;7:117-40.

24. Kimia S Hossein K, Hamid H, et al. Evaluation of Biofilm Formation Among Klebsiella pneumoniae Isolates and Molecular Characterization by ERIC-PCR J undishapur J Microbiol. 2016 ; 9(1):

25. Suman E, Jose J, Varghese S, Kotian M S. Study of biofilm production in Escherichia coli causing urinary tract infection. Indian J Med Microbiol 2007;25:305-6 .

26. Mohan S, Dinesh B, Karthikeyan D. A Study on correlation between the drug resistance and biofilm production among the GNB isolated from blood. Indian J Microbiol Res 2016;3(2):197-202.

*Corresponding author:

Dr. Santosh Kotgire, Department of Microbiology, Indian Institute of Medical Sciences \& Research, Warudi, Jalna, Maharashtra (India)

Phone: +91 9922867658

Email: santosh_kots2001@yahoo.com

Financial or other Competing Interests: None. 